Editor's Note: These short reviews of a recent paper in the Journal, written exclusively by graduate students or postdoctoral fellows, are intended to mimic the journal clubs that exist in your own departments or institutions. For more information on the format and purpose of the Journal Club, please see http://www.jneurosci.org/misc/ifa_features.shtml.

\title{
A New Unified Framework for Making and Implementing Decisions
}

\author{
Sven Bestmann \\ Wellcome Department of Imaging Neuroscience and Sobell Department of Motor Neuroscience and Movement Disorders, University College London, \\ London WC1N 3BG, United Kingdom \\ Review of Cisek (http://www.jneurosci.org/cgi/content/full/26/38/9761)
}

In almost every aspect of life, optimal behavior requires continuous monitoring and processing of incoming sensory stimuli, weighing their contextual relevance, deciding between alternative actions, and selecting the most appropriate behavior. For example, a goalkeeper facing a penalty kick needs to observe player and ball, estimate the likely trajectory of the ball based on experience and postural cues from the player, and finally implement the decision into a fast and accurate action.

Commonly, this sequence is viewed as occurring serially and in distinct brain regions, with activity related to decisionmaking preceding action planning and its implementation. However, both parietal and premotor cortex exhibit decisionrelated as well as sensory- and motorrelated activity (Platt and Glimcher, 1999; Romo et al., 2004). Until recently, such findings have rarely been fully incorporated into models of decision-making and action planning.

In their computational model presented in the The Journal of Neuroscience, Cisek (2006) abandon the idea of decision-making and action planning as serial, spatially distinct processes. Rather, the model assumes that multiple decision alternatives and potential actions occur si-

Received Nov. 1, 2006; revised Nov. 8, 2006; accepted Nov. 8, 2006. Correspondence should be addressed to Sven Bestmann at the above address. E-mail: s.bestmann@fil.ion.ucl.ac.uk.

DOI:10.1523/JNEUROSCI.4758-06.2006

Copyright $\odot 2006$ Society for Neuroscience $\quad$ 0270-6474/06/2613121-02\$15.00/0 multaneously across multiple stages of the cortical hierarchy (Fig. 1).

To test the model, Cisek (2006) implemented an anatomically and electrophysiologically informed neural network. This network comprised a frontoparietal (dorsal) and occipitofrontal stream in which population activity was simulated during visually cued center-out pointing tasks, with and without delays and varying informative cue values. Neurons in the dorsal stream [parietal cortex (PPC) and premotor cortex (PMd)] were directionally selective but insensitive to color, whereas neurons in prefrontal cortex (PFC) were color sensitive with low directional specificity [Cisek (2006), his Fig. 1 (http:// www.jneurosci.org/cgi/content/full/26/ 38/9761/F1) and supplemental material]. Neuronal layers at each node were recurrently interconnected, with excitatory influence between similarly directionally tuned neurons and inhibitory influences between neurons having orthogonal directional tuning. Similar features governed "categorical" neurons in PFC. The model maintains stimulus-specific information even after termination of visual input through bidirectional reinforcing interactions between PPC and PMd and modulatory input from PFC.

Cisek (2006) proposes that frontoparietal population activity is tuned to a range of movement parameters, interacting with incoming sensory and cognitive input. This is similar to the view that population activity encodes a conditional prob- ability density function for potential values of the encoded parameter (here movement direction). Herein, population activity can encode several action alternatives at the same time by assigning valence and precision to them through different magnitude and width of different peaks within that population, respectively [Cisek (2006), his Fig. 2 (http://www. jneurosci.org/cgi/content/full/26/38/9761/ F2)]. Indeed, when tested in a cued delayed-response task, delay-period network activity in both PMd and PPC represented competing target locations, mirroring population activity recorded in monkeys using the same paradigm (Cisek and Kalaska, 2005).

How then does the brain select between these multiple representations for potential actions? Here, the model elegantly borrows from the biased competition mechanism, originally proposed for visual attention (Desimone, 1998). The prediction is that alternative actions across the entire visuomotor hierarchy (rather than only at distinct stages, as implied by serial processing models) are in competition with one another. Without sufficient evidence in favor of one or another action, competing action alternatives coexist. However, increasing bias for one action suppresses activity encoding alternative actions via lateral inhibition, thereby making way for a final response decision. In the model, PFC neurons provide this biasing activity [Cisek (1996), his Fig. 3 (http://www.jneurosci.org/cgi/ 
content/full/26/38/9761/F3)]. Increasing certainty about the required action increases the biasing signal, which increases firing rates in corresponding PMd neurons while suppressing competing PMd activity.

The decision then occurs in a winnertakes-it-all manner, or so-called "quenching threshold" (Grossberg, 1973). As the author points out, this is crucially different from decision threshold models: the "quenching" threshold is constantly weighted by the number of action alternatives, their respective salience, and their angular distance (in the case of spatial decision-making, as here), rather than being a fixed value. A decision is "made" when a quenching threshold is eventually reached in PMd (rather than prefrontal or parietal cortex).

A stunning feature of the model was that its behavior followed the predicted responses across several tasks. For example, in the presence of a single spatial cue, PMd activity represented only a single action: there was no ambiguity about what action to choose. When a categorical color-cue removed uncertainty about the action required after appearance of two spatial targets, initially occurring brief bimodal PMd responses (presumably reflecting the visual input) were almost immediately replaced by unimodal responses that represented the required action. Moreover, the model replicates a finding not accounted for by previous models of decision-making. Not only the number of choices (targets) influences reaction times, but also the spatial distance between them. Spatially nearby targets have overlapping population peaks, and the time taken to reach a quenching threshold is markedly reduced. In general, the model closely captured in vivo electrophysiological (Cisek and Kalaska, 2005) and behavioral reaction time data (Bock and Eversheim, 2000), giving it an additional stamp of credence.

But this is just a first step, and the data raises several interesting questions: First, does the model generalize onto nonspatial decision-making (e.g., value-based decisions)? Second, the model does not incorporate how other premotor regions with direct access to M1 (e.g., ventral premotor cortex and supplementary motor area) may contribute to decision-making for

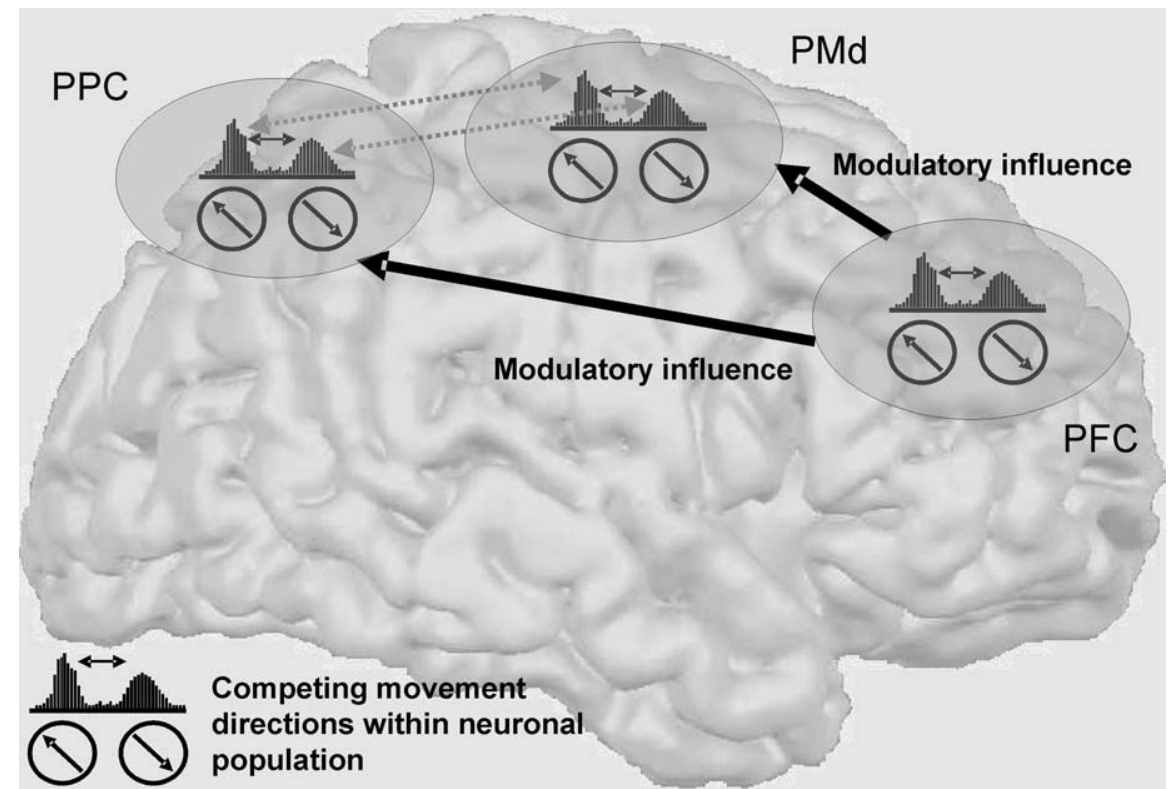

Figure 1. Model schematic illustrating that competing decisions and actions are simultaneously represented within the same population of reciprocally interconnected frontoparietal areas, which receive modulating input from PFC.

actions. Third, the model poses that (spatial) decisions are made in PMd (the moment the quenching threshold is reached). Modulatory PFC signals bias evidence for one or another action, but the model does not yet explain how this signal is actually computed. This contrasts with some existing concepts of decision-making which assign the decision-making process to prefrontal and parietal regions (for review, see Sugrue et al., 2005), and one could argue that in the model by Cisek (2006), the information about decisions is actually fully contained in the biasing PFC signal. Moreover, information about competing decisions and actions may be processed in parallel across multiple cortical nodes, yet the actual decision is still being made in PMd, implying some degree of serial processing.

In principle, these questions can be addressed by model modifications. The use of classical and Bayesian model comparison techniques would then allow for directly comparing alternative and competing hypotheses, an important step for model validation. Although the current model accurately replicates in vivo data, the question is now open about which model may be the most expedient and flexible one.

Nevertheless, the elegance of the model of Cisek (2006) is that it accounts for the correlated activity of both sensorymotor as well as decision variables in many brain regions. This is an important step toward a unifying framework for decision-making and the corresponding selection of actions.

\section{References}

Bock O, Eversheim U (2000) The mechanisms of movement preparation: a precuing study. Behav Brain Res 108:85-90.

Cisek P (2006) Integrated neural processes for defining potential actions and deciding between them: a computational model. J Neurosci 26:9761-9770.

Cisek P, Kalaska JF (2005) Neural correlates of reaching decisions in dorsal premotor cortex: specification of multiple direction choices and final selection of action. Neuron 45:801-814.

Desimone R (1998) Visual attention mediated by biased competition in extrastriate visual cortex. Philos Trans R Soc Lond B Biol Sci 353:1245-1255.

Grossberg S (1973) Contour enhancement, short term memory, and constancies in reverberating neural networks. Studies Appl Math 52:213-257.

Platt ML, Glimcher PW (1999) Neural correlates of decision variables in parietal cortex. Nature 400:233-238.

Romo R, Hernandez A, Zainos A (2004) Neuronal correlates of a perceptual decision in ventral premotor cortex. Neuron 41:165-173.

Sugrue LP, Corrado GS, Newsome WT (2005) Choosing the greater of two goods: neural currencies for valuation and decision making. Nat Rev Neurosci 6:363-375. 